\title{
Use of Nonsteroidal Anti-Inflammatory Drugs and Bladder Cancer Risk: A Meta-Analysis of Epidemiologic Studies
}

\author{
Haifeng Zhang ${ }^{1}$, Dongpeng Jiang ${ }^{2}$, Xuedong $\mathrm{Li}^{{ }^{1}}{ }^{*}$ \\ 1 Department of Urology Surgery, The Second Clinical College, Harbin Medical University, Harbin, Heilongjiang Province, China, 2 Department of Urology Surgery, \\ Heilongjiang Provincial Corps Hospital of the Chinese People's Armed Police Force, Harbin, Heilongjiang Province, China
}

\begin{abstract}
Purpose: Several epidemiologic studies have evaluated the association between nonsteroidal anti-inflammatory drugs (NSAIDs) and bladder cancer risk and the results were varied. Thus, we conducted a comprehensive meta-analysis of studies exclusively dedicated to the relationship between the 3 most commonly used analgesics and bladder cancer risk.

Methods: A systematic literature search up to November 2012 was performed in PubMed database for 3 categories of analgesics: acetaminophen, aspirin or non-aspirin NSAIDs. Study-specific risk estimates were pooled using a random-effects model.

Results: Seventeen studies (8 cohort and 9 case-control studies), involving a total of 10,618 bladder cancer cases, were contributed to the analysis. We found that acetaminophen (relative risk [RR] 1.01, 95\% confidence interval [Cl] 0.88-1.17) and aspirin (RR $1.02,95 \% \mathrm{Cl} 0.91-1.14$ ) were not associated with bladder cancer risk. Although non-aspirin NSAIDs was statistically significantly associated with reduced risk of bladder cancer among case-control studies (but not cohort studies), the overall risk was not statistically significant (RR $0.87,95 \% \mathrm{Cl} 0.73-1.05$ ). Furthermore, we also found that non-aspirin NSAIDs use was significantly associated with a $43 \%$ reduction in bladder cancer risk among nonsmokers (RR $0.57,95 \%$ C 0.43-0.76), but not among current smokers.

Conclusion: The results of our meta-analysis suggest that there is no association between use of acetaminophen, aspirin or non-aspirin NSAIDs and bladder cancer risk. However, non-aspirin NSAIDs use might be associated with a reduction in risk of bladder cancer for nonsmokers.
\end{abstract}

Citation: Zhang H, Jiang D, Li X (2013) Use of Nonsteroidal Anti-Inflammatory Drugs and Bladder Cancer Risk: A Meta-Analysis of Epidemiologic Studies. PLoS ONE 8(7): e70008. doi:10.1371/journal.pone.0070008

Editor: Bharat B. Aggarwal, The University of Texas M. D. Anderson Cancer Center, United States of America

Received April 6, 2013; Accepted June 14, 2013; Published July 19, 2013

Copyright: (C) 2013 Zhang et al. This is an open-access article distributed under the terms of the Creative Commons Attribution License, which permits unrestricted use, distribution, and reproduction in any medium, provided the original author and source are credited.

Funding: These authors have no support or funding to report

Competing Interests: The authors have declared that no competing interests exist.

*E-mail: lixuedong123@hotmail.com.

\section{Introduction}

Bladder cancer is the most common malignant tumor of the urinary system. According to the International Agency for Research on Cancer for 2008, about 386,300 individuals were diagnosed with bladder cancer and 150,200 died as a result. The majority of bladder cancer occurs in males and the highest incidence rates are found in the countries of Europe, North America, and Northern Africa [1]. In the US, bladder cancer is the fourth most common cause of cancer among men and the ninth most common cause of cancer death among men [2]. The overall public health importance of bladder cancer is increasing with the growing elderly population.

Cigarette smoking and occupational exposures are the main risk factors for bladder cancer in Western countries, whereas chronic infection with Schistosoma hematobium in developing countries accounts for about $50 \%$ of the total burden [3]. Other environmental factors, including selenium intake [4], chlorination by-products [5] and low dose arsenic levels in drinking water [6], have also been associated with bladder cancer, but are less wellestablished.

Nonsteroidal anti-inflammatory drugs (NSAIDs) are among the most frequently used drugs worldwide. Experimental and epidemiologic evidence strongly suggests that aspirin and non-aspirin NSAIDs have shown promise as chemopreventive agents [7]. Most epidemiologic studies have reported inverse associations between NSAIDs use and the risk of breast [8], gastric [9], and colorectal cancer [10]. However, whether NSAIDs use may reduce the risk of bladder cancer remains unclear. There have been few metaanalyses of NSAIDs use and cancer risk in general, which included some studies of bladder cancer and did not exclusively focus on this disease [11]. The effect of NSAIDs on the risk of bladder cancer remains to be determined. Therefore, we conducted a comprehensive meta-analysis of studies exclusively dedicated to the relationship between the 3 most commonly used analgesics and bladder cancer risk. 


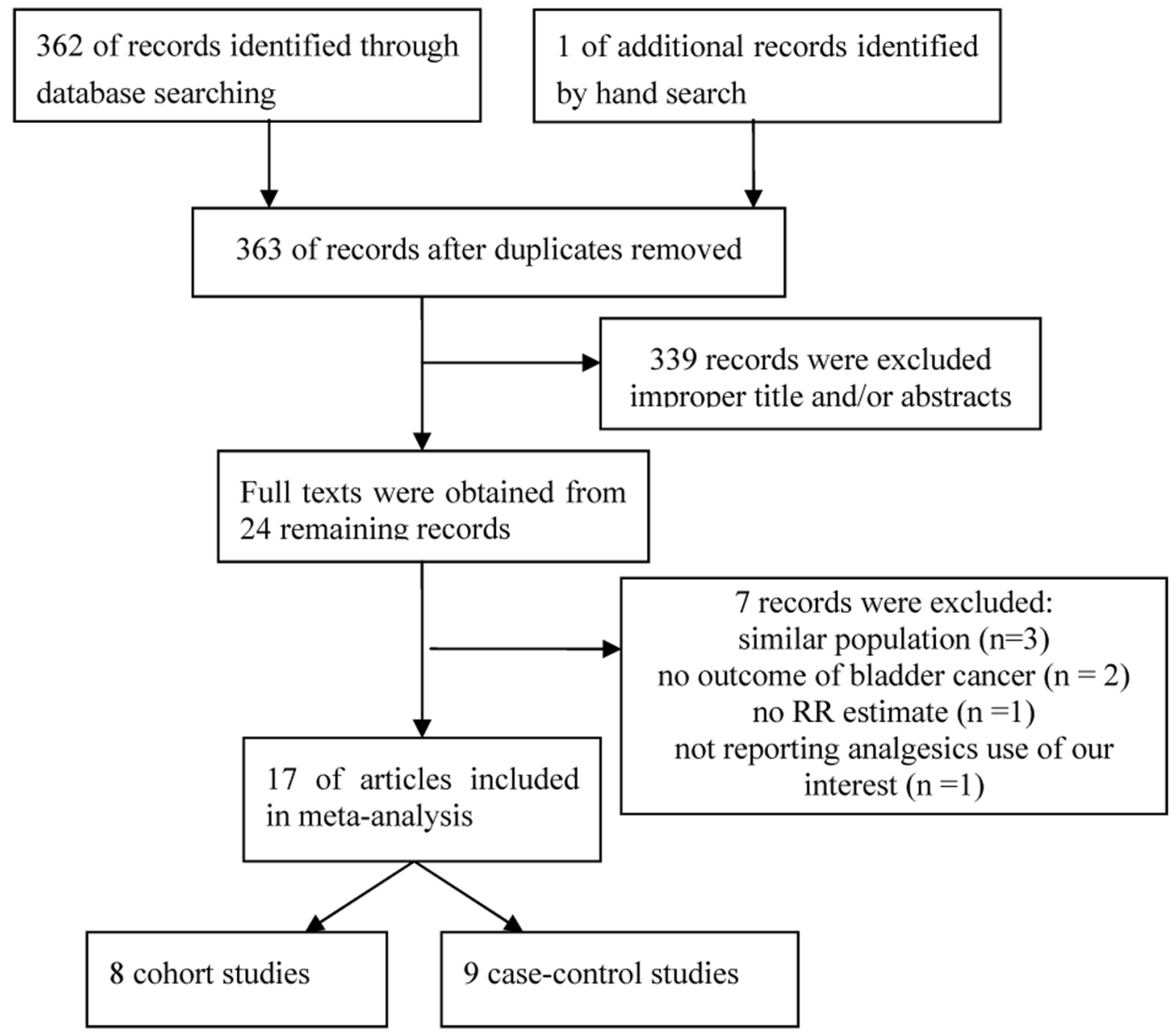

Figure 1. Flow diagram of study identification.

doi:10.1371/journal.pone.0070008.g001

\section{Materials and Methods}

\section{Search Strategy}

A systematic literature search up to November 1 of 2012 was performed in PubMed database to identify eligible studies. Search terms included "acetaminophen," "aspirin," "nonsteroidal antiinflammatory agents," or "NSAID" combined with "bladder cancer," "bladder neoplasms," or "bladder carcinoma". The titles and abstracts of the studies identified in the search were scanned to exclude any clearly irrelevant studies. The full texts of the remaining articles were read to determine whether they contained information on the topic of interest. Furthermore, we also manually searched the reference lists of every article retrieved and review papers to find any additional published studies. All searches were conducted independently by 2 authors (HZ and DJ). The results were compared, and any questions or discrepancies were resolved through iteration and consensus.

\section{Study Selection}

To be eligible, studies had to fulfill the following 4 inclusion criteria: 1) had a case-control or prospective study design; 2) reported results on aspirin, non-aspirin NSAIDs or acetaminophen use; 3) the outcome was bladder cancer incidence or mortality; and 4) reported the estimate of relative risk (RR) with their corresponding 95\% confidence interval (CI) (or sufficient data to calculate of these effect measure). Studies reporting different measures of RR like risk ratio, rate ratio, hazard ratio (HR), and odds ratio $(\mathrm{OR})$ were included in the meta-analysis. In practice, these measures of effect yield a similar estimate of RR, since the absolute risk of bladder cancer is low.

\section{Data Extraction}

Data abstraction was conducted independently by 2 researchers ( $\mathrm{HZ}$ and $\mathrm{DJ}$ ), with disagreements resolved by consensus. The following information were collected: the first author's last name, year of publication, country in which the study was performed, study design, years of follow-up or the study period, study participants age range, number of subjects and number of bladder cancer cases, used drugs, exposure definition, information source, control of confounding factors by matching or adjustment, and RR estimates with corresponding 95\% CIs. If a study provided several risk estimates, the most completely adjusted estimate was extracted. Differences in data extraction were resolved by consensus, referring back to the original article.

\section{Statistical analysis}

Separate analyses were performed according to use of acetaminophen, aspirin, and non-aspirin NSAIDs. Study-specific risk estimates were extracted from each article, and log risk estimates were weighted by the inverse of their variances to obtain a pooled risk estimate. We pooled study-specific log RRs to 
Table 1. Characteristics of studies included in the meta-analysis.

\begin{tabular}{|c|c|c|c|c|c|c|c|c|c|c|}
\hline Study & Year & Country & Design & $\begin{array}{l}\text { Study } \\
\text { period }\end{array}$ & Age, y & $\begin{array}{l}\text { N. of } \\
\text { participant }\end{array}$ & BC Cases & Drug(s) & $\begin{array}{l}\text { Information } \\
\text { source }\end{array}$ & Adjustments* \\
\hline Piper (23) & 1985 & USA & C-C & 1975-1979 & $20-49$ & 173 & 173 & ACE & Interview & $1-3$ \\
\hline Derby (24) & 1996 & USA & $C-C$ & 1980-1991 & $\geq 20$ & 2,009 & 504 & ACE & Database & $1,2,4,5$ \\
\hline Pommer (25) & 1999 & Germany & C-C & 1990-1994 & 70.4 & 921 & 571 & $\mathrm{ACE} / \mathrm{ACA}$ & Interview & $1,6-8$ \\
\hline Castelao (26) & 2000 & USA & $C-C$ & 1987-1996 & $25-74$ & 1514 & 1514 & $\mathrm{ACE} / \mathrm{ACA}$ & Interview & $1,2,6,9,10$ \\
\hline Kaye (27) & 2001 & USA & $\mathrm{C}-\mathrm{C}$ & 1995-1998 & $>50$ & 187 & 744 & ACE & Database & $1,2,6,11,12,13,14$ \\
\hline Friis (28) & 2002 & Denmark & Co & 1989-1995 & 63 & 39,946 & 115 & ACE & Database & 1,2 \\
\hline Friis (29) & 2003 & Denmark & Co & 1989-1997 & 70 & 29,470 & 161 & ASA & Database & 1,2 \\
\hline Sørensen (30) & 2003 & Denmark & Co & 1989-1995 & 47.2 & 172,057 & 330 & NA-NSAIDs & Database & 1,2 \\
\hline Ratnasinghe (31) & 2004 & USA & Co & 1971-1992 & $25-74$ & 22,843 & 40 & ASA & Interview & $2,6,7,9,14,15$ \\
\hline Blumentals (32) & 2004 & USA & $C-C$ & 1992-1994 & 71.1 & 1,293 & 330 & NA-NSAIDs & Database & $1,2,6,16$ \\
\hline Fortuny(33) & 2006 & Spain & C-C & $1997-2000$ & $20-80$ & 1,029 & 958 & ACE/ACA/NA-NSAIDs & Interview & $1,2,3,6,16$ \\
\hline Fortuny (34) & 2007 & USA & $\mathrm{C}-\mathrm{C}$ & 1998-2001 & $25-74$ & 463 & 376 & $\mathrm{ACE} / \mathrm{ACA}$ & Interview & $1,2,6,16$ \\
\hline Genkinger (35) & 2007 & USA & Co & 1986-2004 & $40-75$ & 49,448 & 607 & ACE/ACA & Questionnaire & $1,3,6,17,18$ \\
\hline Daugherty (36) & 2011 & USA & Co & 1993-2005 & 62.1 & 508,842 & 2,489 & ASA/NA-NSAIDs & Questionnaire & $6,14-16,19$ \\
\hline Jacobs (37) & 2012 & USA & Co & $1997-2008$ & NR & 100,139 & 150 & ASA & Questionnaire & $1,2,6,9,14-16,20-26$ \\
\hline Shih (38) & 2012 & USA & Co & $2000-2010$ & $50-76$ & 77,048 & 385 & ASA/NA-NSAIDs & Questionnaire & $1,2,6,9,15,16,27$ \\
\hline Baris (39) & 2013 & USA & $C-C$ & 2001-2004 & $30-79$ & 1,418 & 1,171 & ACE/ACA/NA-NSAIDs & Interview & $1,2,3,6,15,28$ \\
\hline
\end{tabular}

Abbreviations: BC, bladder cancer; C-C, case control; Co, cohort; NR, not reported; ACE: Acetaminophen; ASA: aspirin; NA-NSAIDs: nonaspirin NSAIDs.

*1, age; 2 , sex; 3 , residence; 4 , certain occupations; 5 , coffee drinking; 6 , smoking; 7 , socioeconomic status; 8 , laxative intake, 9 , education; 10 , number of years employed as hairdresser/barber, 11, general practice; 12, duration of prescription history in the database; 13, index date; 14, body mass index, 15, race; 16, analgesic use; 17, period; 18 , fluid intake; 19 , study; 20 , physical activity; 21 , history of heart disease; 22 , stroke; 23 , diabetes; 24 , hypertension; 25 , cholesterol-lowering drug use; 26 , history of colorectal endoscopy; 27, family history of bladder cancer; 28 , Hispanic status.

doi:10.1371/journal.pone.0070008.t001

compute an overall RR and its 95\% CI for regular/any use versus reference group from each study. For reference group, it was defined as "subjects who never took analgesics or who were not regular takers". Where data for different intake levels or different duration of use were available, we subsequently restricted the analyses to the highest intake or the longest duration given by each study. Studies were combined by using the DerSimonian and

Table 2. Exposure definition in each study.

\begin{tabular}{|c|c|}
\hline Study & Exposure definition \\
\hline Piper (23) & Regular use (daily use for at least 30 days per year) vs. no use \\
\hline Derby (24) & Any use ( $\geq 1$ prescription in past year) vs. no use \\
\hline Pommer (25) & Regular use (lifelong cumulative amount of $\geq 1 \mathrm{~kg}$ ) vs. no use \\
\hline Castelao (26) & Regular use ( $\geq 2$ times a week for $\geq 1$ month) vs. no/irregular use \\
\hline Kaye (27) & Any use ( $\geq 1$ prescription) vs. no use \\
\hline Friis (28) & Any use( $\geq 1$ prescription) vs. no use \\
\hline Friis (29) & Regular use low-dose aspirin (75-150 mg once daily) vs. no use \\
\hline Sørensen (30) & Any use ( $\geq 1$ prescription) vs. no use \\
\hline Ratnasinghe (31) & Any use (use any aspirin in past 30 days or 6 months ) vs. no use \\
\hline Blumentals (32) & Any use ( $\geq 1$ prescription) vs. no use \\
\hline Fortuny(33) & Regular use ( $\geq 2$ times a week for $\geq 1$ month) vs. no use \\
\hline Fortuny (34) & Regular use ( $\geq 4$ times a week for $\geq 1$ month) vs. no use \\
\hline Genkinger (35) & Regular use ( $\geq 2$ times a week) vs. no use \\
\hline Daugherty (36) & Regular use ( $>2$ times a week) vs. no use \\
\hline Jacobs (37) & Regular use (daily use) vs. no use \\
\hline Shih (38) & Regular use ( $>1$ tine a week for $\geq 1$ year) vs. no use \\
\hline Baris (39) & Regular use ( $\geq 2$ times a week for $\geq 1$ month) vs. no use \\
\hline
\end{tabular}


Study

(reference)

$\mathrm{RR}(95 \% \mathrm{Cl})$

Case-control

Piper et al., 1985 (23)

Derby and Jick 1996 (24)

Pommer et al., 1999 (25)

Castelao et al.,2000 (26)

Kaye et al., 2001 (27)

Fortuny et al., 2006(33)

Fortuny et al., 2007 (34)

Baris et al., 2013 (39)

Subtotal (I-squared $=26.0 \%, p=0.221$ )

Cohort

Friis et al., 2002 (28)

Genkinger et al., 2007 (35)

Subtotal $\quad(I-$ squared $=0.0 \%, p=0.773)$

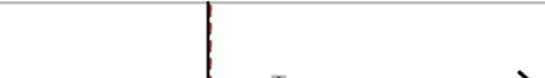

$1.50(0.40,7.20)$

$1.30(0.60,2.80)$

$0.83(0.33,2.07)$

$0.85(0.60,1.19)$

$0.91(0.64,1.31)$

$0.80(0.40,1.30)$

$0.70(0.40,1.20)$

$1.30(1.10,1.70)$

$0.99(0.82,1.20)$

$1.00(0.70,1.50)$

$0.90(0.49,1.65)$

$0.97(0.70,1.34)$

$1.01(0.88,1.17)$

Overall $\quad($ I-squared $=7.3 \%, p=0.375)$

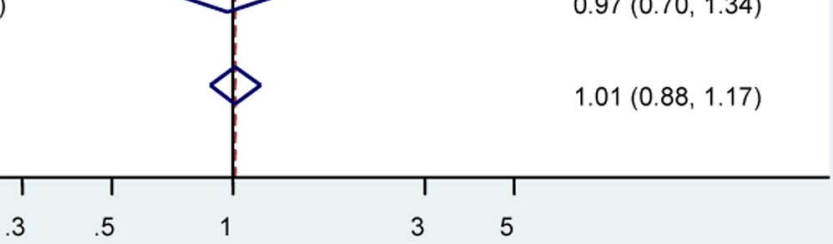

Figure 2. Risk estimates of bladder cancer associated with regular/any use of acetaminophen. Squares indicate study-specific risk estimates (size of the square reflects the study-specific statistical weight, i.e., the inverse of the variance); horizontal lines indicate $95 \%$ confidence intervals (Cls); diamonds indicate summary risk estimate with its corresponding $95 \%$ confidence interval. doi:10.1371/journal.pone.0070008.g002

Laird random-effects model, which considers both within- and between-study variations [12].

Statistical heterogeneity among studies was assessed using the Cochrane's Q statistic, and inconsistency was quantified with the $I^{2}$ statistic that estimates the percentage of total variation across studies due to heterogeneity rather than chance [13]. For the $\mathrm{Q}$ statistic, a $P$ value $<0.10$ was considered statistically significant for heterogeneity; for $I^{2}$, a value $>50 \%$ is considered a measure of severe heterogeneity. When statistical heterogeneity was detected, sensitivity analyses were performed. Publication bias was evaluated with Egger's regression test in which $P$ value less than 0.10 was considered representative of statistically significant publication bias [14]. All statistical analyses were performed with Stata 10 software (Stata Corporation, College Station, Texas). We performed this meta-analysis in accordance with the guidelines of the Preferred Reporting Items for Systematic Reviews and Meta-analyses (PRISMA) statement [15].

\section{Results}

\section{Literature Search}

The detailed steps of our literature search are shown in Figure 1. Briefly, our initial search strategy retrieved a total of 363 citations. After the titles and abstracts were screened, 339 articles were excluded because they were laboratory studies, review articles, or irrelevant to the current study. We identified 24 potentially relevant articles. Three articles were excluded because they reported on similar population [16-18]. Two publications were excluded because there were no outcomes of bladder cancer $[19,20]$, one was excluded because it did not provide RR estimate [21] and the remaining one was excluded because it did not report analgesics use of our interest [22]. Finally, 17 articles [23-39] were included in this meta-analysis (Figure 1).

The 17 relevant studies were published between 1985 and 2012, including 8 cohort studies [28-31,35-38] and 9 case-control studies [23-27,32-34,39]. A total of $1,008,800$ participants, including 10,618 bladder cancer cases were involved in these studies and followed for 3-21 years. Ten studies were used for analysis of acetaminophen use [23-28,33-35,39], 11 for aspirin use [25,26,29,31,33-39] and 6 for non-aspirin NSAIDs use $[30,32,33,36,38,39]$. The characteristics of the included studies for the 3 most commonly used analgesics are summarized in Table 1. Most studies provided risk estimates that were adjusted for age (16 studies), sex (14 studies) and smoking (12 studies); fewer were adjusted for race ( 6 studies), body mass index (4 studies), and education (4 studies). The exposure definitions of the included studies are shown in Table 2.

\section{Acetaminophen}

The multivariable-adjusted RRs of bladder cancer for regular/ any use of acetaminophen in individual observational studies and summary estimate are shown in Figure 2. Regular/any use of 
Table 3. Summary risk estimates.

\begin{tabular}{|c|c|c|c|c|c|c|}
\hline \multirow[t]{2}{*}{ Stratification group } & \multirow[t]{2}{*}{ References } & \multicolumn{2}{|c|}{ RR $(95 \% \mathrm{Cl})$} & \multicolumn{3}{|c|}{ Heterogeneity test } \\
\hline & & & & $Q$ & $P$ & $r^{2}(\%)^{\dagger}$ \\
\hline \multicolumn{7}{|l|}{ Acetaminophen } \\
\hline Regular/any use & $23-28,33-35,39$ & 1.01 & $0.88-1.17$ & 9.70 & 0.375 & 7.3 \\
\hline Case-control studies & $23-27,33,34,39$ & 0.99 & $0.82-1.20$ & 9.46 & 0.221 & 26.0 \\
\hline Cohort studies & 28,35 & 0.97 & $0.70-1.34$ & 0.08 & 0.773 & 0 \\
\hline High intake & $24,26-28,33,39$ & 1.08 & $0.81-1.44$ & 3.98 & 0.552 & 0 \\
\hline Long duration & $33,34,39$ & 0.80 & $0.43-1.48$ & 3.53 & 0.171 & 43.4 \\
\hline US & $23,24,26,27,34,35,39$ & 1.01 & $0.82-1.23$ & 8.63 & 0.195 & 30.5 \\
\hline Europe & $25,28,33$ & 0.92 & $0.68-1.25$ & 0.45 & 0.800 & 0 \\
\hline \multicolumn{7}{|l|}{ Aspirin } \\
\hline Regular/any use & $25,26,29,31,33-39$ & 1.02 & $0.91-1.14$ & 19.49 & 0.035 & 48.7 \\
\hline Case-control studies & $25,26,33,34,39$ & 0.95 & $0.76-1.18$ & 9.94 & 0.042 & 59.7 \\
\hline Cohort studies & $29,31,35-38$ & 1.06 & $0.93-1.20$ & 8.51 & 0.130 & 41.2 \\
\hline High intake & $26,33,35,36,38,39$ & 0.96 & $0.83-1.12$ & 7.04 & 0.218 & 29.0 \\
\hline Long duration & $33,34,37,39$ & 1.04 & $0.82-1.32$ & 0.93 & 0.819 & 0 \\
\hline Men & $29,31,36$ & 1.10 & $0.99-1.21$ & 2.08 & 0.354 & 3.7 \\
\hline Women & $29,31,36$ & 1.43 & $0.68-3.00$ & 13.12 & 0.001 & 84.8 \\
\hline No smoker & $35,36,38$ & 0.90 & $0.75-1.09$ & 0.24 & 0.885 & 0 \\
\hline Current smoker & $35,36,38$ & 0.99 & $0.81-1.21$ & 0.04 & 0.982 & 0 \\
\hline US & $26,31,34-39$ & 0.97 & $0.84-1.12$ & 16.06 & 0.025 & 56.4 \\
\hline Europe & $25,29,33$ & 1.16 & $0.99-1.33$ & 0.83 & 0.662 & 0 \\
\hline \multicolumn{7}{|l|}{ Non-aspirin NSAIDs } \\
\hline Regular/any use & $30,32,33,36,38,39$ & 0.87 & $0.73-1.05$ & 24.19 & $<0.001$ & 79.3 \\
\hline Case-control studies & $32,33,39$ & 0.76 & $0.62-0.95$ & 3.19 & 0.203 & 37.3 \\
\hline Cohort studies & $30,36,38$ & 0.98 & $0.78-1.22$ & 11.87 & 0.003 & 83.1 \\
\hline No smoker & $32,36,38$ & 0.57 & $0.43-0.76$ & 0.04 & 0.979 & 0 \\
\hline Current smoker & 36,38 & 1.24 & $0.63-2.46$ & 2.31 & 0.129 & 56.7 \\
\hline US & $32,36,38,39$ & 0.86 & $0.79-0.94$ & 2.19 & 0.534 & 0 \\
\hline Europe & 30,33 & 0.74 & $0.25-2.15$ & 7.96 & 0.005 & 87.4 \\
\hline
\end{tabular}

Abbreviation: $\mathrm{RR}$, relative risk; $\mathrm{Cl}$, confidence intervals.

${ }^{\dagger} / 2$ is interpreted as the proportion of total variation across studies that are due to heterogeneity rather than chance.

doi:10.1371/journal.pone.0070008.t003

acetaminophen was not associated with the risk of bladder cancer (RR 1.01, 95\% CI 0.88-1.17). The Cochran's Q test resulted in a $P=0.38(\mathrm{Q}=9.70)$, and the corresponding quantity $I^{2}$ was $7.3 \%$, both indicating that the results of those studies were homogeneous. The $P$ value for the Egger test was $P=0.23$, suggesting a low probability of publication bias. The associations of acetaminophen use with bladder cancer risk did not differ by study type (Table 3 ).

Further, six studies [24,26-28,33,39] were reported RR estimates of the association between high intake of acetaminophen and bladder cancer risk (Table 3). Based on the results from those studies, the calculated combined RR for bladder cancer in high intake of acetaminophen was found to be 1.08 (95\% CI $0.81-$ 1.44). And the association was also not significant (RR 0.80, 95\% CI 0.43-1.48) with long duration of acetaminophen use among limited number of studies with that available information $[33,34,39]$. Stratified analysis by country did not show any statistically significant difference in summary estimates between strata.

\section{Aspirin}

The multivariable-adjusted RRs of bladder cancer for regular/ any use of aspirin in individual observational studies and summary estimate are shown in Figure 3. The pooled RR of bladder cancer for regular/any aspirin use was 1.02 (95\% CI 0.91-1.14). There was statistically significant heterogeneity among studies $(P=0.035$, $\left.I^{2}=48.7 \%\right)$. The Egger test showed no evidence of publication bias for aspirin $(P=0.686)$. The associations of aspirin use with bladder cancer risk did not differ by study type (Table 3 ).

To explore the heterogeneity among studies of aspirin use and bladder cancer, we performed sensitivity analyses. By using a stepwise process, we determined that most of heterogeneity was accounted for the study by Fortuny et al [34]. After excluding this single study, there was no study heterogeneity $(P=0.20$, $I^{2}=26.4 \%$ ), and the RR was essentially unchanged (RR 1.05, 95\% CI 0.96-1.15).

High intake or long duration of aspirin use was also not associated with bladder cancer risk (Table 3). In addition, stratified analysis by smoking status, gender and country did not show any 


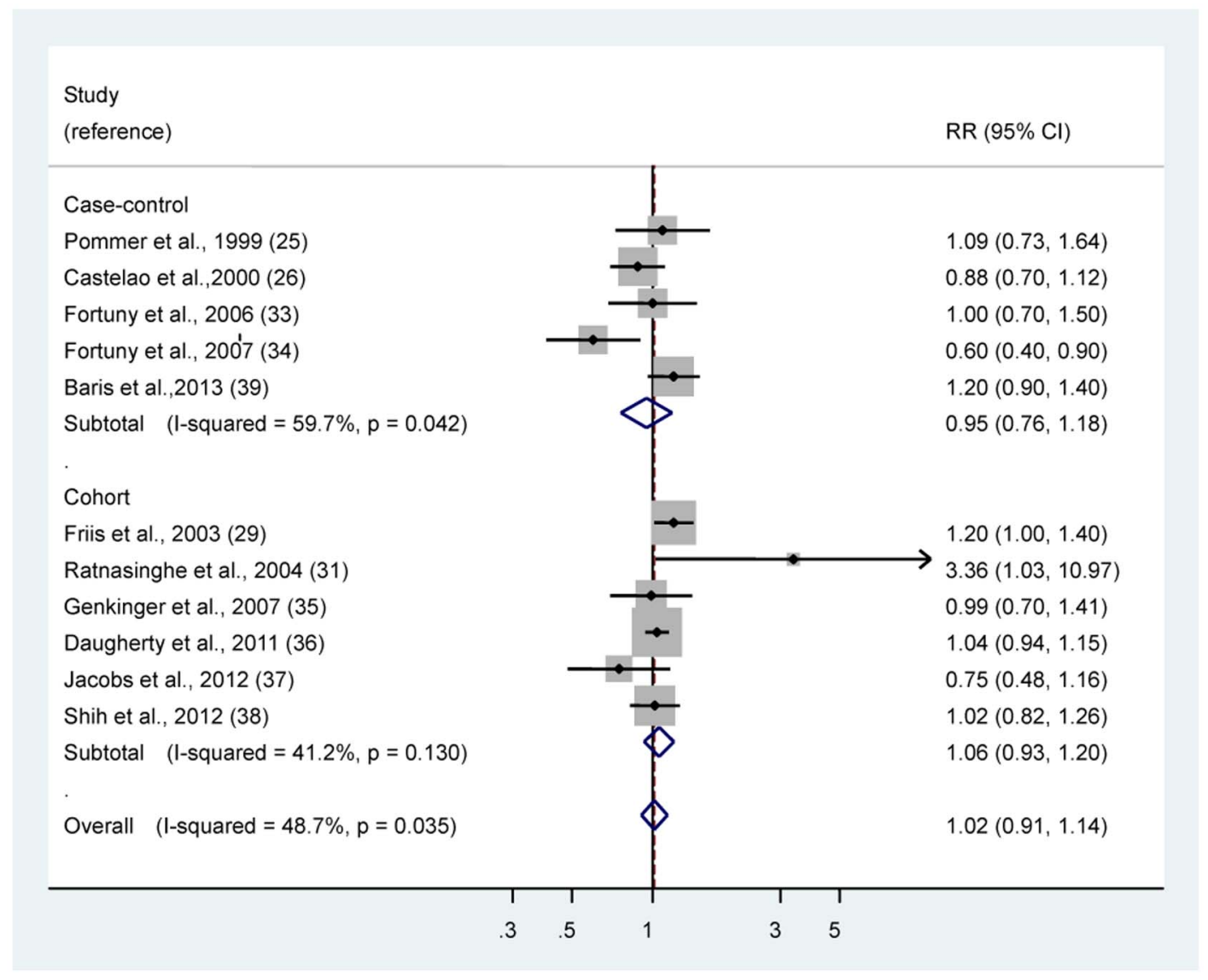

Figure 3. Risk estimates of bladder cancer associated with regular/any use of aspirin. Squares indicate study-specific risk estimates (size of the square reflects the study-specific statistical weight, i.e., the inverse of the variance); horizontal lines indicate $95 \%$ confidence intervals (Cls); diamonds indicate summary risk estimate with its corresponding $95 \%$ confidence interval. doi:10.1371/journal.pone.0070008.g003

statistically significant difference in summary estimates between strata.

\section{Non-aspirin NSAIDs}

Six studies [30,32,33,36,38,39] were reported RR estimates of the association between non-aspirin NSAIDs use and bladder cancer risk (Figure 4). No association was observed between regular/any non-aspirin NSAIDs use and the risk of bladder cancer (RR 0.87, 95\% CI 0.73-1.05). There was statistically significant heterogeneity among studies $\left(P<0.001, I^{2}=79.3 \%\right)$. The Egger test showed no evidence of publication bias for aspirin $(P=0.118)$. And we found that non-aspirin NSAIDs use was statistically significantly associated with reduced risk of bladder cancer among case-control studies, but not among cohort studies (Table 3).

To explore the heterogeneity, we also performed the sensitivity analyses. By using a stepwise process, we determined that most of heterogeneity was accounted for the study by Sørensen et al [30]. After excluding this single study, there was no study heterogeneity $\left(P=0.19, I^{2}=34.7 \%\right)$, and a significant association was observed (RR 0.83 , 95\% CI $0.74-0.94$ ).

In addition, stratified analysis by smoking status found that nonaspirin NSAIDs use was statistically significantly associated with $43 \%$ reduced risks of bladder cancer among nonsmokers (RR 0.57; 95\% CI, 0.43-0.76), but not among current smokers (RR $1.24 ; 95 \%$ CI, 0.63-2.46) (Table 3). And we found that nonaspirin NSAIDs use was statistically significantly associated with reduced risk of bladder cancer among studies from the US, but not among studies from Europe.

\section{Discussion}

This present meta-analysis included 17 studies $(8$ cohort and 9 case-control studies), involving a total of 10,618 bladder cancer cases. In this meta-analysis of the 3 most commonly used analgesics and bladder cancer risk, we found that acetaminophen and aspirin were not associated with bladder cancer risk. Although non-aspirin NSAIDs was statistically significantly associated with reduced risk of bladder cancer among case-control studies (but not cohort studies), the overall risk was not statistically significant. Also, non-aspirin NSAIDs was significantly associated with a $43 \%$ reduction in bladder cancer risk among nonsmokers.

We found that acetaminophen use was not associated with the risk of bladder cancer with a pooled $\mathrm{RR}=1.01$ from 10 studies. Acetaminophen is a metabolite of phenacetin, a well-known banned carcinogen which has been more so linked to renal pelvic tumor [40]. Recently, a meta-analysis found that acetaminophen use was associated with $21 \%$ reduced risk of kidney cancer and the risk was higher with higher intake. In this meta-analysis, we did not found the associations of long term and high dose of acetaminophen use with bladder cancer risk.

In our meta-analysis, we did not found that aspirin use was associated with bladder cancer risk from 11 studies. Our results are in agreement with a large randomized controlled trial [41]. In the 
Study

(reference)

$\mathrm{RR}(95 \% \mathrm{Cl})$

\section{Cohort}

Sørensen et al., 2003 (30)

Daugherty et al., 2011 (36)

Shih et al., 2012 (38)

Subtotal $\quad(I-$ squared $=83.1 \%, p=0.003$ )

Case-control

Blumentals et al., 2004 (32)

Fortuny et al., 2006 (33)

Baris et al.,2013 (39)

Subtotal $\quad(I-$ squared $=37.3 \%, p=0.203$ )
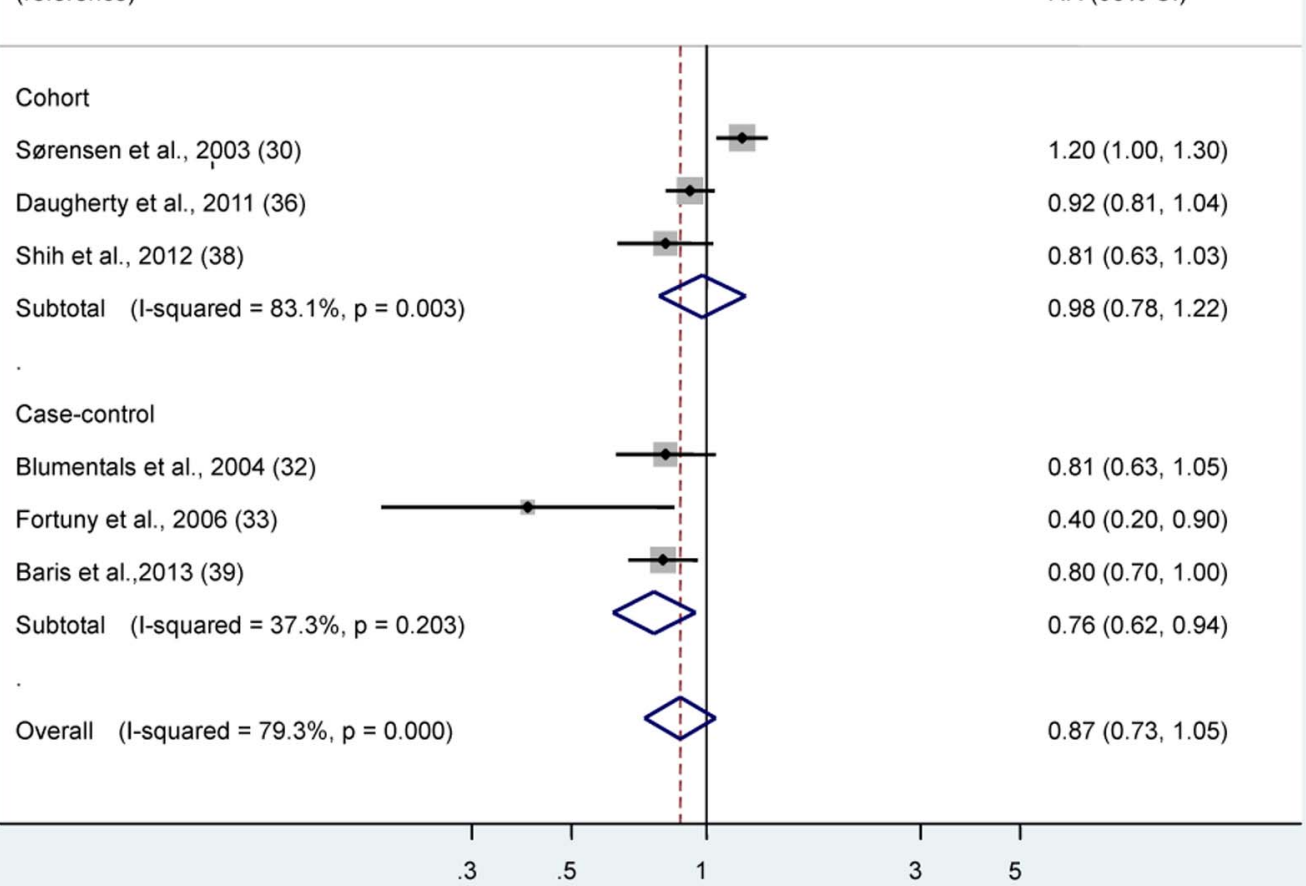

$1.20(1.00,1.30)$

$0.92(0.81,1.04)$

$0.81(0.63,1.03)$

$0.98(0.78,1.22)$

$0.81(0.63,1.05)$

$0.40(0.20,0.90)$

$0.80(0.70,1.00)$

$0.76(0.62,0.94)$

$0.87(0.73,1.05)$

3.5

Figure 4. Risk estimates of bladder cancer associated with regular/any use of non-aspirin NSAIDs. Squares indicate study-specific risk estimates (size of the square reflects the study-specific statistical weight, i.e., the inverse of the variance); horizontal lines indicate $95 \%$ confidence intervals (Cls); diamonds indicate summary risk estimate with its corresponding $95 \%$ confidence interval.

doi:10.1371/journal.pone.0070008.g004

Women's Health Study, low dose aspirin (100 mg every other day) for an average of ten years did not lower bladder cancer incidence. And in the meta-analysis of aspirin and cancer risk, the pooled risk for bladder cancer was 0.95 (95\% CI 0.83-1.07) [11].

We found from 6 studies that non-aspirin NSAIDs use was not associated with the risk of bladder cancer in general. Nevertheless, there was significant heterogeneity by study. After excluding the study by Sørensen et al [30], a significant inverse association was found. In this record linkage study from Denmark with 330 bladder cancer cases, prescribed non-aspirin NSAIDs slightly increased the risk of bladder cancer (RR 1.2; 95\% CI, 1.0-1.3), although no dose-response relationship was observed. Misclassification is likely in studies relying solely on prescription data as many commonly used NSAIDs do not require a prescription. Therefore, due to the limited published information and relatively small number of cases, we still cannot draw the firm conclusion about non-aspirin NSAIDs use and bladder cancer risk. Further studies with a larger number of subjects may be able to discriminate the effects of specific NSAIDs more clearly.

Furthermore, an interest finding in our meta-analysis, although more limited, is that smoking status might modify the association of use of nonaspirin NSAIDs with bladder cancer risk. Smoking is a well-known risk factor for bladder cancer. There are over 60 carcinogens, including nitrosamines, aromatic amines, and polycyclic aromatic hydrocarbons, have been detected in cigarette smoke. Metabolic activation of these carcinogens leads to the formation of DNA adducts, causing miscoding and other mutations [42]. While smokers have been shown to have increased cycloxygenase (COX)-2 expression and activity in their urothelial tissues [43], it may be that the anticarcinogenic effects of nonaspirin NSAIDs against COX-2 are overwhelmed by the carcinogenic effects of smoking. Thus, a decrease in bladder cancer risk associated with nonaspirin NSAIDs has been shown in nonsmokers in our meta-analysis.

Our study has several strengths: it is the most up-to-date comprehensive review of analgesics on one specific type of cancer, bladder cancer. It includes the 3 mostly used contemporary drugs and 17 observational studies were included in our meta-analysis, reporting data of more than 1 million participants, including 10,618 bladder cancer patients. Meta-analysis of studies with large numbers of incident cases provides high statistical power for estimating the relationship between exposure and outcome risk. Moreover, in a meta-analysis of published studies, publication bias could be of concern since small studies with null results tend not to be published. In this meta-analysis, however, we found little evidence of publication bias.

Nevertheless, several limitations are worth mentioning. First, a meta-analysis is not able to solve problems with confounding factors that could be inherent in the included studies. Inadequate control for confounders may bias the results in either direction, toward exaggeration or underestimation of risk estimates. However, most observational studies in this meta-analysis adjusted for other known and potential risk factors for bladder cancer. Second, heterogeneity may be introduced because of methodologic and demographic differences among studies. We used appropriate well-motivated inclusion criteria to maximize homogeneity, and 
performed sensitivity and subgroup analyses to investigate potential sources of heterogeneity. Third, studies used different definition of analgesic use, which might have limited comparability of the results across the studies. However, our findings were stable and robust in the subgroup analyses. Fourth, long-term analgesic users may switch the type of analgesics they use over time. Because almost all of the studies assessed analgesics use at baseline only, we were not able to evaluate the impact of change of use of analgesics. In addition, the study does not clearly distinguish between multiple exposures to analgesics. The possibility of confounding would be especially relevant for long duration users, as those patients could have used phenacetin many years previously. Last, because of lack of data, it was not possible to address the important issues of dose and duration of use needed to achieve effects.

\section{References}

1. Jemal A, Bray F, Center MM, Ferlay J, Ward E, et al. (2011) Global cancer statistics. CA Cancer J Clin 61: 69-90.

2. Siegel R, Naishadham D, Jemal A (2012) Cancer statistics, 2012. CA Cancer J Clin 62: 10-29.

3. Parkin DM (2006) The global health burden of infection-associated cancers in the year 2002. Int J Cancer 118: 3030-3044.

4. Zeegers MP, Kellen E, Buntinx F, van den Brandt PA (2004) The association between smoking, beverage consumption, diet and bladder cancer: a systematic literature review. World J Urol 21: 392-401.

5. Villanueva CM, Fernandez F, Malats N, Grimalt JO, Kogevinas M (2003) Meta-analysis of studies on individual consumption of chlorinated drinking water and bladder cancer. J Epidemiol Community Health 57: 166-173.

6. Guo HR, Chiang HS, Hu H, Lipsitz SR, Monson RR (1997) Arsenic in drinking water and incidence of urinary cancers. Epidemiology 8: 545-550.

7. Harris RE, Beebe-Donk J, Doss H, Burr Doss D (2005) Aspirin, ibuprofen, and other non-steroidal anti-inflammatory drugs in cancer prevention: a critical review of non-selective COX-2 blockade (review). Oncol Rep 13: 559-583.

8. Luo T, Yan HM, He P, Luo Y, Yang YF, et al. (2012) Aspirin use and breast cancer risk: a meta-analysis. Breast Cancer Res Treat 131: 581-587.

9. Tian W, Zhao Y, Liu S, Li X (2010) Meta-analysis on the relationship between nonsteroidal anti-inflammatory drug use and gastric cancer. Eur J Cancer Prev 19: 288-298.

10. Rostom A, Dubé C, Lewin G, Tsertsvadze A, Barrowman N, et al (2007) Nonsteroidal anti-inflammatory drugs and cyclooxygenase-2 inhibitors for primary prevention of colorectal cancer: a systematic review prepared for the U.S. Preventive Services Task Force. Ann Intern Med 146: 376-389.

11. Bosetti C, Rosato V, Gallus S, Cuzick J, La Vecchia C (2012) Aspirin and cancer risk: a quantitative review to 2011. Ann Oncol 23: 1403-1415.

12. DerSimonian R, Laird N (1986) Meta-analysis in clinical trials. Control Clin Trials 7: 177-188.

13. Higgins JP, Thompson SG (2002) Quantifying heterogeneity in a meta-analysis. Stat Med 21: 1539-1558.

14. Egger M, Davey Smith G, Schneider M, Minder C (1997) Bias in meta-analysis detected by a simple, graphical test. BMJ 315: 629-634

15. Moher D, Liberati A, TetzlaffJ, Altman DG (2009) Preferred reporting items for ystematic reviews and meta-analyses: the PRISMA statement. Ann Intern Med 51: 264-269, W264.

16. Schreinemachers DM, Everson RB (1994) Aspirin use and lung, colon, and breast cancer incidence in a prospective study. Epidemiology 5: 138-146.

17. Thun MJ, Namboodiri MM, Calle EE, Flanders WD, Heath CW Jr (1993) Aspirin use and risk of fatal cancer. Cancer Res 53: 1322-1327.

18. Jacobs EJ, Thun MJ, Bain EB, Rodriguez C, Henley SJ (2007) A large cohort study of long-term daily use of adult-strength aspirin and cancer incidence. J Natl Cancer Inst 99: 608-615.

19. Steineck G, Wiholm BE, Gerhardsson de Verdier M (1995) Acetaminophen, some other drugs, some diseases and the risk of transitional cell carcinoma. A population-based case-control study. Acta Oncol 34: 741-748.

20. Rosenberg L, Rao RS, Palmer JR, Strom BL, Zauber A, et al (1998) Transitional cell cancer of the urinary tract and renal cell cancer in relation to acetaminophen use (United States). Cancer Causes Control 9: 83-88.

21. Paganini-Hill A, Chao A, Ross RK, Henderson BE (1989) Aspirin use and chronic diseases: a cohort study of the elderly. BMJ 299: 1247-1250.

22. Langman MJ, Cheng KK, Gilman EA, Lancashire RJ (2000) Effect of antiinflammatory drugs on overall risk of common cancer: case-control study in general practice research database. BMJ 320: 1642-1646.

23. Piper JM, Tonascia J, Matanoski GM (1985) Heavy phenacetin use and bladder cancer in women aged 20 to 49 years. N Engl J Med 313: 292-295.
In summary, the results of this meta-analysis of 17 observational studies indicated that use of acetaminophen, aspirin or non-aspirin NSAIDs was not associated with bladder cancer risk. However, non-aspirin NSAIDs use might be associated with a reduction in risk of bladder cancer for nonsmokers.

\section{Supporting Information}

\section{Checklist S1 PRISMA Checklist for the meta-analysis. (DOC)}

\section{Author Contributions}

Conceived and designed the experiments: HZ XL. Performed the experiments: HZ DJ XL. Analyzed the data: HZ DJ. Contributed reagents/materials/analysis tools: HZ DJ. Wrote the paper: HZ XL.

24. Derby LE, Jick H (1996) Acetaminophen and renal and bladder cancer. Epidemiology 7: 358-362.

25. Pommer W, Bronder E, Klimpel A, Helmert U, Greiser E, et al. (1999) Urothelial cancer at different tumour sites: role of smoking and habitual intake of analgesics and laxatives. Results of the Berlin Urothelial Cancer Study. Nephrol Dial Transplant 14: 2892-2897.

26. Castelao JE, Yuan JM, Gago-Dominguez M, Yu MC, Ross RK (2000) Nonsteroidal anti-inflammatory drugs and bladder cancer prevention. Br J Cancer 82: 1364-1369.

27. Kaye JA, Myers MW, Jick H (2001) Acetaminophen and the risk of renal and bladder cancer in the general practice research database. Epidemiology 12: 690 694.

28. Friis S, Nielsen GL, Mellemkjaer L, McLaughlin JK, Thulstrup AM, et al. (2002) Cancer risk in persons receiving prescriptions for paracetamol: a Danish cohort study. Int J Cancer 97: 96-101.

29. Friis S, Sørensen HT, McLaughlin JK, Johnsen SP, Blot WJ, et al. (2003) A population-based cohort study of the risk of colorectal and other cancers among users of low-dose aspirin. Br J Cancer 88: 684-688.

30. Sørensen HT, Friis S, Nørgård B, Mellemkjaer L, Blot WJ, et al. (2003) Risk of cancer in a large cohort of nonaspirin NSAID users: a population-based study. Br J Cancer 88: 1687-1692.

31. Ratnasinghe LD, Graubard BI, Kahle L, Tangrea JA, Taylor PR et al. (2004) Aspirin use and mortality from cancer in a prospective cohort study. Anticancer Res 24: 3177-3184.

32. Blumentals WA, Foulis PR, Schwartz SW, Mason TJ (2004) Analgesic therapy and the prevention of bladder cancer. Urol Oncol 22: 11-15.

33. Fortuny J, Kogevinas M, Garcia-Closas M, Real FX, Tardón A, et al. (2006) Use of analgesics and nonsteroidal anti-inflammatory drugs, genetic predisposition, and bladder cancer risk in Spain. Cancer Epidemiol Biomarkers Prev 15: 16961702.

34. Fortuny J, Kogevinas M, Zens MS, Schned A, Andrew AS, et al. (2007) Analgesic and anti-inflammatory drug use and risk of bladder cancer: a population based case control study. BMC Urol 7: 13.

35. Genkinger JM, De Vivo I, Stampfer MJ, Giovannucci E, Michaud DS (2007) Nonsteroidal antiinflammatory drug use and risk of bladder cancer in the health professionals follow-up study. Int J Cancer 120: 2221-2225.

36. Daugherty SE, Pfeiffer RM, Sigurdson AJ, Hayes RB, Leitzmann M, et al. (2011) Nonsteroidal antiinflammatory drugs and bladder cancer: a pooled analysis. Am J Epidemiol 173: 721-730.

37. Jacobs EJ, Newton CG, Gapstur SM, Thun MJ (2012) Daily aspirin use and cancer mortality in a large US cohort. J Natl Cancer Inst 104: 1208-1217.

38. Shih C, Hotaling JM, Wright JL, White E (2012) Long-term NSAID use and incident urothelial cell carcinoma in the VITamins and Lifestyle (VITAL) study. Urol Oncol. [Epub ahead of print]

39. Baris D, Karagas MR, Koutros S, Colt JS, Johnson A, et al. (2013) Nonsteroidal anti-inflammatory drugs and other analgesic use and bladder cancer in northern New England. Int J Cancer 132: 162-173.

40. Clissold SP (1986) Paracetamol and phenacetin. Drugs 32: 46-59.

41. Cook NR, Lee IM, Gaziano JM, Gordon D, Ridker PM, et al (2005) Low-dose aspirin in the primary prevention of cancer: the Women's Health Study: a randomized controlled trial. JAMA 294: 47-55.

42. Pfeifer GP, Denissenko MF, Olivier M, Tretyakova N, Hecht SS, et al (2002) Tobacco smoke carcinogens, DNA damage and p53 mutations in smokingassociated cancers. Oncogene 2002;21: 7435-7451.

43. Badawi AF, Habib SL, Mohammed MA, Abadi AA, Michael MS (2002 Influence of cigarette smoking on prostaglandin synthesis and cyclooxygenase-2 gene expression in human urinary bladder cancer. Cancer Invest 20: 651-656. 